\title{
Oxidative stress effects in the uterus, placenta and fetus of pregnant rats submitted to acute and chronic stress ${ }^{1}$
}

\begin{abstract}
Adriano Silva Silveira', Ricardo Dutra Aydos", Rondon Tosta Ramalho'I', landara Schettert Silvalv, Ruy de Araujo Caldas", Abílio Torres dos Santos Netov", Camila Tozaki Rodrigues ${ }^{\mathrm{VII}}$

'Fellow Master degree, Postgraduate Program in Health and Development in the Midwest Region, Universidade Federal do Mato Grosso do Sul (UFMS), Campo Grande-MS, Brazil. Conception and design of the study; technical procedures; acquisition, interpretation and analysis of data; manuscript writing.

"PhD, Associate Professor of Surgery, Postgraduate Program in Health and Development in the Midwest Region, UFMS, Campo Grande-MS, Brazil. Conception and design of the study, interpretation of data, manuscript writing, critical revision, final approval.

I'Full Professor, Laboratory of Experimental Carcinogenicity, UFMS, Campo Grande-MS, Brazil. Macroscopic and histopathologic analysis.

IVFull Professor, Postgraduate Program in Health and Development in the Midwest Region, UFMS, Campo Grande-MS, Brazil. Intellectual and scientific content of the study.

vSenior Visiting Professor, UFMS, Campo Grande-MS, Brazil. English language, manuscript writing.

${ }^{V}$ 'Fellow Master degree, Postgraduate Program in Health and Development in the Midwest Region, UFMS, Campo Grande-MS, Brazil. Technical procedures.

VIINurse, UFMS, Campo Grande-MS, Brazil. Design of the study, technical procedures.
\end{abstract}

\section{Abstract}

Purpose: To evaluate the effects of oxidative stress in pregnant rats submitted to acute and chronic stress, relating to alterations in the uterus, placenta and fetus.

Methods: Twenty-four female Wistar albino (Rattus norvegicus), were divided into four groups, for induction of oxidative stress the animals were submitted to cold and physical immobilization. Plasma fasting glucose and MDA were determined in all groups and the fetuses and placentas were measured.

Results: There were no statistical differences in the levels of malonic dialdehyde (MDA), however the averages of chronic stress group were higher compared to control groups, which could explain the observed adverse effects; there was no correlation between puppies' size, the weight of the placenta and MDA values.

Conclusions: Chronic stress causes adverse effects, when compared to control groups; chronic stress group had fetuses, placentas and number of puppies, significantly lower compared to other groups. The rats exposed to chronic stress, also presented a higher frequency of fetal resorption.

Key words: Oxidative Stress. Lipid Peroxidation. Rats. 


\section{Introduction}

Reactive oxygen species (ROS) generating in oxidative stress promotes physiological and pathological changes in the female reproductive tract. ROS are detected in the ovaries, uterine tube and embryos which are involved in several physiological processes, such as oocyte maturation, steroidogenesis and corpus luteum ${ }^{1}$.

Concerning infertility, it is known that steroidogenesis is related to antioxidant concentration. High concentrations of estrogen contribute to a greater enzymatic antioxidant activity; furthermore oxidative stress has an impact on the production of steroid hormones produced by granulosa cells and that the oocyte within the follicle is naturally exposed to oxidative stress leading to lipid peroxidation and influencing the production glycoproteins produced by the granulosa cells ${ }^{2}$.

Oxidative stress causes embryos injury may due to peroxidation of membrane phospholipids and chemical modifications of different types of biomolecules. The consequences of these damages include mitochondrial activity, embryonic development and apoptosis. Oocytes and embryos are protected from oxidative stress by the presence of antioxidants from follicular and oviduct fluids ${ }^{3}$.

One of the theories considered is related to decreased uteroplacental flow, which leads to poor fetal oxygenation. This effect is caused by the inadequate invasion of the intravascular trophoblast, which maintains the high resistance of the spiral arteries consequently endothelial injury, elevation of reactive oxygen species (ROS) and reactive nitrogen species (RNS). Low antioxidant capacity of placental tissue leads the development of oxidative stress ${ }^{4}$.

The oxidative stress is a consequence of imbalance in the amount of reactive oxygen species (ROS), commonly called free radicals.
This imbalance may be caused by a number of factors like inadequate nutrition and animal's retention in stressful conditions related to higher ROS production and/or reduced antioxidant activity.

Experimental models should provide data for both biological and behavioral phenomena to understand the pathological processes that apply to the human being 5 .

The most important experimental models described in the literature are those of induced or spontaneous disease. To understand pathophysiological mechanisms of diseases, effects of medications and surgical procedures thus experimental animal models can be used in all fields of biological research, they are very important for medical science. Trials in humans are limited and a series of procedures are required for ethical reasons to preserve physical and psych emotional integrity. In addition, when a study of an entire population becomes a limiting factor, experimental animal models can provide data statistically comparable to the whole population based on the concept of sampling, since they represent a portion of it ${ }^{5,6}$.

Studies of lipid peroxidation in pregnancy are limited to determine the etiology and pathophysiology of the factors that trigger oxidative stress, animal models have been proposed for understanding the physiology and pathology when is impossible to control variables in humans. Many of these experimental models use the induction of chronic or acute physical stress to establish and understand relationships between stressor events. It has been reported that stressors elevate blood pressure and the risk for preeclampsia. The stress is associated with complications during pregnancy, premature birth and intrauterine growth restriction ${ }^{7,8}$.

Rat's models are appropriate to understand the mentioned stressors because of the similarity of the placenta and its trophoblastic invasion between rats and humans ${ }^{9}$. 
In the placental uterus process, it is assumed that oxidative stress is a causal factor in chronic complications and can be measured based on the level of thiobarbituric acid reactive substances (TBARS) and catalase activity $^{10}$

Arguably, the most important event at the onset of normal placentation is the establishment of effective maternal circulation, a process that is linked to the physiological conversion of maternal spiral arteries into a flaccid sinoid duct with low resistance that allows the blood supply necessary to the development of the placenta ${ }^{11}$.

In contrast, the fetal origin trophoblastic invasion process contributes to the transformation of the uteroplacental circulation into a high capacity, low resistance system that exhibits relative resistance to vasopressor agents ${ }^{12}$.

This process occurs in humans in two stages. Initially, the deciduous segments of the spiral arteries are affected by the first wave of trophoblastic invasion followed by a second wave, placental ischemia. Progresses continuously during pregnancy and the narrowed vessels do not provide the demand for the developing placental fetal unit, a hypoxia insurge, generating oxidative stress and strong inflammatory response of maternal origin. There is increased production of placental antiangiogenic factors, specifically soluble forms of tyrosine kinase 1 (sFLT-1) and soluble endoglin ${ }^{13}$.

Other factors such as alterations in the renin-angiotensin-aldosterone axis, poor immune adaptation, excessive loss of trophoblasts fragments and genetic factors seem to contribute to the pathogenesis of abnormal placentation ${ }^{12,22}$.

The oxidative stress is in other words, an imbalance between the pro and antioxidant species, and the antioxidant species are at disadvantage $\mathrm{e}^{13,14}$.
Basically, there are two ways to measure oxidative stress, by evaluating the concentrations of both by-products and promoters of the oxidative damage; which provides the dimensions of the damage; and by the antioxidant capacity of the medium in response to oxidative stress which determines the adaptation the organism to the unfavorable environment ${ }^{13,15}$.

The most commonly used markers for assessing lipid damage by oxidation are the MDA test (the main metabolite of peroxilipid breakdown), which uses the TBARS assay ${ }^{13}$. This test identifies lipid oxidation products, bile pigments, amino acids and sugars that can generate interfering chromogens. In view of this, it is more appropriate to use the term thiobarbituric acid reactive substances (TBARS). Moreover, the fact that TBA is calibrated with MDA leads the authors to express their results with the term "amount of MDA"16.

Gestational diabetes were used induced in rats to study different mechanisms that have been proposed for the increase in the production of free radicals in the hyperglycemic state, among them glucose metabolism as the main source of $\mathrm{ROS}^{17,18}$ the lipid peroxidation induced by hyperglycemia ${ }^{19}$ and its advanced glycosylation end -products (AGEs), which, through their cellular receptors, modify and inactivate enzymes ${ }^{20}$, stimulate the production of $\mathrm{ROS}^{21}$, as well the transcriptional activity of NF-KB, a classically inflammatory pathway ${ }^{23}$. Thus, chronic hyperglycemia, through oxidative stress, functionally compromises cellular proteins, membrane phospholipids (formation of malondialdehyde) and nucleic acids, in addition to stimulating the production of inflammatory cytokines such as tumor necrosis factor alpha (TNF- $\alpha)^{8,16}$, events strongly related to the development of diabetic comorbidities.

The consequences on the body weight of the fetus or neonates of diabetic rats depend not only on the level of glycemia 
during pregnancy but also on the life period studied. It has been shown in rodents that intrauterine exposure to low doses of STZ during pregnancy is associated with normal weight or macrosomia 25,26 . On the other hand, studies carried out with the offspring of mothers submitted to high doses of STZ showed that the animals had reduced growth in adulthood ${ }^{27}$.

Newborns, especially preterm infants, are highly susceptible to tissue and organ damage by free radicals, notably those derived from oxygen, with the superoxide anion being the most important of the reactive oxygen intermediates (ROI) and produced in response to hypoxic stimuli - ischemic or inflammatory. In addition, gestation itself is a physiological condition with increased metabolic demand and increased tissue oxygen requirements and, in the event of abnormalities in pregnancy, oxidative imbalance may occur, and excess free radicals can promote damage to the fetus, which already have an antioxidant defense system, it is important to emphasize that the main intracellular antioxidant component, glutathione, only shows its maximum production at the end of gestation ${ }^{28-30}$.

In this work, the effects of oxidative stress (lipid peroxidation) in pregnant rats submitted to acute and chronic stress related to changes in the uterus, placenta and fetuses were determined.

\section{- Methods}

The study was approved by the Animals Research Ethics Committee of UFMS (protocol CEUA / UFMS number 647/2014) and was performed in accordance with the International Guiding Principles for Biomedical Research Involving Animals, published by the Council for International Organizations of Medical Sciences (CIOMS), as well as with the Brazilian law on the scientific use of animals (Law 11794/2008).
Adult female Wistar rats 45 days old, weighing 150-200 grams, were obtained from the Central Laboratory for Animals (UFMS), were randomized into four groups: non-pregnant rats without stress $(G 1, n=6)$, control pregnant rats $(G 2, n=6)$, pregnant rats subjected to acute stress $(G 3, n=6)$ and pregnant rats subjected to cronic stress (G4, $n$ =6).

The animals were housed in a polypropylene cage with containing paddy husk as bedding. Each cage of G2, G3 and G4 maintained a ratio of three females to one male, except in $\mathrm{G} 1$, which did not contain male in the cage (Figure 1).

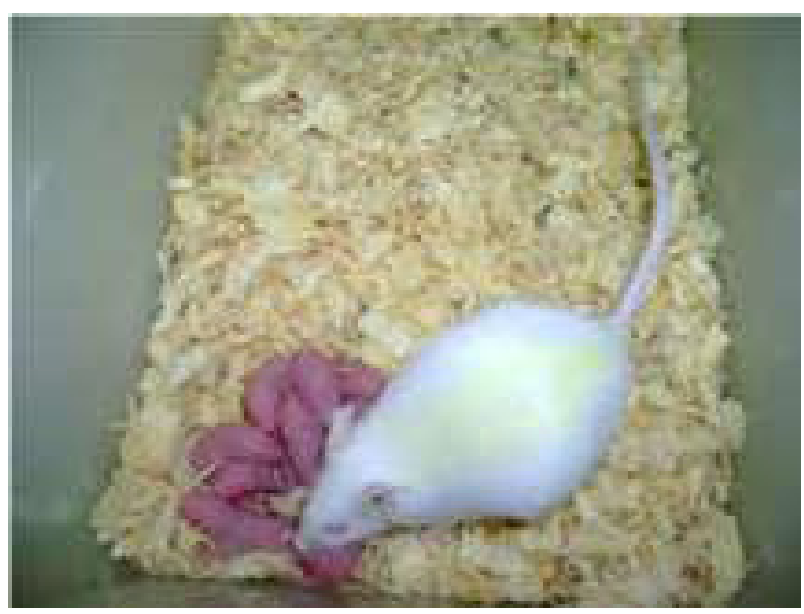

Figure 1 - Female with their cubs on the day of childbirth / euthanasia.

All animals were kept in ventilated rack and maintained at controlled conditions of temperature $\left(23 \pm 1^{\circ} \mathrm{C}\right)$, humidity .and dark-light cycle (12 hours). The animals had free access to standard pellet (Nuvilab) and water ad libitum.

The mating was performed and the first day of pregnancy was considered the third day of stay of the male in the cage.

For the induction of clinical and laboratory of oxidative stress in animals, the model of exposure to cold and physical immobilization was used. In G3, the physical immobilization was carried out with a $15 \times 7 \mathrm{~cm}$ 
carton for 5 minutes on the 7th and 14th day of pregnancy. The G4 was kept in a separately ventilated shelf to be exposed to cold. Two liters of liquid nitrogen were used, kept in a semi-open styrofoam box inside the shelf, from the 7th day of pregnancy, in order to maintain the temperature at $+21^{\circ} \mathrm{C}$. The polystyrene box with nitrogen was changed every day until the end of the experiment and the temperature of the ventilated shelf was monitored.

The rats were manipulated during the twenty days of pregnancy/ non-pregnancy and evaluated at $0,7,14$ and 20 days when euthanasia was performed.

\section{Oxidative stress damage}

Malondialdehyde (MDA) levels were measured using thiobarbituric acid reactive substances (TBARS).

Maternal capillary glycemia, fetal weight and size and euthanasia

Fasting blood glucose dosage was performed on days $0,7,14$ and 20 of pregnancy and on non-pregnancies in the equivalent period. Blood collection was performed on the animal's tail and the glycaemia determined utilizing the Accu-Chek ${ }^{\circledR}$ Active blood glucose monitoring system.

Fetuses and placentas were weighed on a QUIMIS ${ }^{\circ}$ precision scale. The size of newborns was measured with a ruler graduated in centimeters (Figure 2).

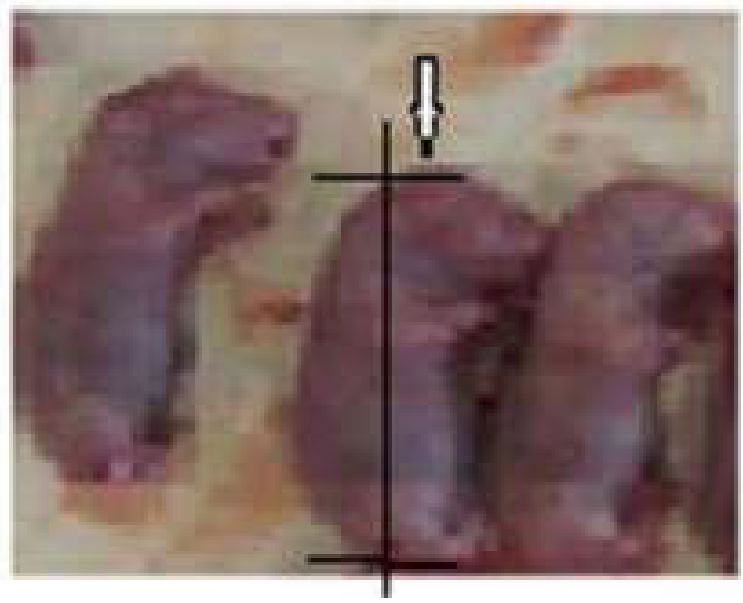

Figure 2 - Cephalo-anal measurement and fetal weighing performed in all groups.

Allanimalsweresubmitted toeuthanasia on the 20th day using intraperitoneal injection with a combination of Ketamine (150mg / kg) and Xylazine $(75 \mathrm{mg} / \mathrm{kg})$. Death was confirmed after cardiac puncture exsanguination.

\section{Statistical analysis}

The data were tabulated in Excel version 2013. For the discrimination of the difference between groups, the Mann-Whitney $U$ test was used followed by Student's t-test. The Pearson correlation test was performed on MDA variables, fetal weight, fetal cephalal-anal size and placental weight. The other results evaluated in this study were described in tables and graphs. The software used was BioEstat 5.0, using $p<0.05$

\section{- Results}

Table 1 - MDA values, showing oxidative stress, according to groups.

\begin{tabular}{llll}
\hline & \multicolumn{3}{c}{ MDA } \\
\hline GNC & GPC & GPEA & GPEC \\
$158.92 \pm 108.39$ & $252.77 \pm 243.70$ & $412.38 \pm 468.11$ & $629.81 \pm 329.31$ \\
\hline
\end{tabular}


Table 2 - Average glycemia of females, in $\mathrm{mg} / \mathrm{dl}$ according to group.

\begin{tabular}{lllll} 
& D1 & D7 & D14 & D21 \\
\hline GNC & $99.50 \pm 14.56$ & $97.50 \pm 9.59$ & $80.67 \pm 12.69$ & $136.50 \pm 7.85$ \\
GPC & $98.50 \pm 8.96$ & $111.83 \pm 7.25$ & $90.17 \pm 13.01$ & $80.83 \pm 21.45$ \\
GPEA & $91.83 \pm 10.83$ & $127 \pm 7.77$ & $84.67 \pm 3.72$ & $95.00 \pm 8.76$ \\
GPEC & $95.67 \pm 4.41$ & $96.50 \pm 13.03$ & $85.33 \pm 6.12$ & $88.00 \pm 8.10$ \\
\hline
\end{tabular}

Table 3 - Mean values according to weight, height, placenta weight and number of fetuses/female.

\begin{tabular}{llllc} 
Group & $\begin{array}{l}\text { newborn } \\
\text { weight }\end{array}$ & $\begin{array}{l}\text { newborn } \\
\text { stature }\end{array}$ & $\begin{array}{l}\text { Placenta } \\
\text { weight }\end{array}$ & $\begin{array}{l}\text { Relationship between } \\
\text { fetus and mother }\end{array}$ \\
\hline FPC & $3.92 \pm 2.48$ & $3.90 \pm 1.62$ & $0.50 \pm 0.19$ & $12.60 \pm 2.07$ \\
FPEA & $4.30 \pm 1.38$ & $4.05 \pm 0.87$ & $0.64 \pm 0.20$ & $14.17 \pm 1.33$ \\
FPEC & $3.22 \pm 0.74$ & $3.21 \pm 0.59$ & $0.55 \pm 0.24$ & $10.60 \pm 2.88$ \\
\hline
\end{tabular}

\section{- Discussion}

Oxidative stress and impact of the stressful stimulus on maternal parameters

The average chronic group G4 $(629.81 \mathrm{ng} / \mathrm{ml})$ was the largest and among the G3 Group (412.37ng/ml) and G4 difference, showing that chronic stress causes adverse effects, when compared to control groups (Table1).

By studying the oxidative stress in pregnant women with preeclampsia it was found a reduced antioxidant enzyme activity, however did not identify difference in plasma levels of $\mathrm{MDA}^{27}$. Increase in oxidative status of pregnancy without complications must be accompanied by increased ability of pregnant women to overcome the effects of oxidative stress without pathological consequences ${ }^{18}$. Despite the non-significant difference between acute and chronic stress groups, the average levels of MDA chronic group was higher. Studies that have applied the exercise as a stressor, showed that acute exercise increases oxygen consumption due to mobilization of energy caused by the acute physical stress, leading to increased formation of ERO. Second bear and about 2 to $5 \%$ of the oxygen used in the mitochondria for cellular respiration are converted into free radicals ${ }^{28}$. While the chronic exercise reduces basal levels of damage, increases resistance to oxidative stress, since the body tends to adapt to stressful situations ${ }^{7}$

Evaluation of maternal glucose levels $(\mathrm{mg} / \mathrm{dl})$ of the groups

There was a significant difference between 7 and 20 days, and in the $7^{\text {th }}$ day the capillary glycaemia of the acute stress group (G3) was higher than the non-pregnant (G1) and chronic stress (G4). At the end of the experiment, the glycemia values of G1 and G3 were higher than the control pregnant group (G2) (Table 2).

In this study there was no increase in MDA, which may explain the non-alteration of glycemia in the studied groups.

Although there was no hyperglycemia at the time studied, there was a significant difference in the capillary glycemia of the acute stress group on control and chronic stress.

The rats in this study showed similar values of capillary glycaemia, which sought to establish reference values of biochemical, physiological and morphological parameters in Wistar rats. The values detected in this study were considered normal and reported the implications of the chronicity of oxidative stress on the etiological process of diabetes 
mellitus $^{28}$. The reverse process can occur, i.e. diabetes increases the oxidative stress, reduces the antioxidant system of pregnant rats and can still be related to occurrence of hypertension and abortion ${ }^{13}$.

In this study there was no increase in MDA levels, which may explain normal glycaemia in all groups. The females in the chronic stress group had hyper glycaemia statistically significant differences this can be explained by the fact that stress, fight-or-flight phase (first phase) mobilizes large amount of glucose and oxygen essential to organs. Cortisol liberated into the blood stream inhibits the release of insulin and stimulates processes such as gluconeogenesis, glycogenolysis and lipolysis for energy production. When the stressor stimulus becomes chronic, excessive and continuous production of cortisol leads to fat deposit due to the continuous process of catabolism for energy production. During pregnancy glucose is the main energetic source for the fetus; therefore there is an increasing demand for glucose during pregnancy, explaining the higher glycemic index in chronic stress group compared with the control did not pregnant $^{30}$.

\section{Stress stimulus on fetal-placental parameters}

The mean litter size of the rats was lower in group 2 and the mean of the acute stress group (G3) was higher. Regarding the number of fetal resorption, the chronic stress group was higher.

\section{Average weight of puppies}

The mean litter size of the rats was lower in group 2 and the mean of the acute stress group (G3) was higher. Regarding the number of fetal resorption, the chronic stress group was higher.

The weight of the rats subjected to chronic stress was significantly different when compared to the offspring of acute stress group and control. The off springs of acute stress group did not have different weight in the control group. There was no correlation between fetal weight and MDA level.

Evaluation of oxidative stress was carried out in the laboratory of experimental Carcinogenesis after cardiac puncture; blood was collected in pediatric vacuum tube with EDTA and centrifuged. Determination of plasma concentration of malondialdehyde (MDA) was performed through the MDA reaction with thiobarbituric acid (TBAR), forming the complex MDA-TBA in the ratio of $1: 2$. The tubes were heated in a water bath at $94^{\circ} \mathrm{C}$ for 1 hour and 15 minutes, after cooling were homogenized with 4 $\mathrm{ml}$ of n-butyl alcohol in a Vortex (QL-901) for 30 seconds, following centrifugation at 3,000 rpm for 20 minutes and the supernatants were read at $532 \mathrm{~nm}$ in a Spectrophotometer(LMR-96, Loccus Company). For the calculation of concentration of MDA, was used an equation obtained by standard curve of absorbance. The concentrations expressed in $\mathrm{ng} / \mathrm{ml}$.

Results $=F c \times A b s$, and $F c=4406 / A B S \times$ MDA

All animals were euthanized on day 20 of pregnancy/ non-pregnant by intraperitoneal Ketamine injection $(150 \mathrm{mg} / \mathrm{kg})$ and Xylazine (75 mg/kg) for the removal of the fetus, placenta. The death was confirmed after exsanguination by cardiac puncture, followed by removal of the uterus and in the case of ferns, hypothermia.

The statistical analysis the data were tabulated in Excel version 2013 programme. For the discrimination of the difference between groups was used the Mann-Whitney $\mathrm{U}$ test followed by the Student's t-test. C test was done.

The puppies' size of chronic stress group was statistically lower than those of other groups. There was no difference between the size of the puppies of chronic stress and 
control. There was no correlation between size of the puppies and MDA of rats.

The placenta weight of the acute stress group showed a statistically significant difference in comparison to both the control group and the chronic stress group. There was no correlation between the weight of the placenta and levels of MDA. It was possible to observe a correlation between fetus weight and placenta weight. There were identified a minor fetal weight of the group that was exposed to chronic stress, as well as the findings in this survey, where it was observed that chronic stress group had fetuses, placentas and number of puppies, significantly lower in relation to other groups ${ }^{30}$. Rats exposed to chronic stress, also presented a higher frequency of fetal resorption, which was also observed. The inadequate trophoblastic invasion mechanisms lead to reduced perfusion and placental failure causing intrauterine growth restriction and if there is severe vasoconstriction leads to fetal distress and death ${ }^{27}$ (Table 3).

\section{Conclusions}

Pregnant rats with regard to malondialdehyde presented the highest levels in the group that suffered chronic stress, as well as blood glucose levels when compared to non-pregnant and acute stress groups.

It was observed that chronic stress Group had fetuses, placentas and number of pups, significantly lower compared to other groups. The rats exposed to chronic stress, also presented a higher frequency of fetal resorption.

The weight of the puppies subjected to chronic stress was lower when compared to the acute stress and control group, however the acute stress group showed no weight difference in relation to the control group. There was no correlation between the fetus weights with the levels of MDA.
The fetus size, the chronic stress groups were statistically lower than those of other groups. There was no difference between the size of the puppies of chronic stress and control. There was no correlation between size of the puppies and MDA of rats.

\section{References}

1. Amaral WT, Peraçoli JC. Fatores de risco relacionados à pré-eclâmpsia. Com Ciênc Saúde. 2011;(22 Supl 1):S161-8.

2. Caldas JPS, Vilela MS, Braghini C, Mazzola TN, Marba STM. Uso materno antenatal de corticosteroide e marcadores de estresse oxidativo e de inflamação no sangue de cordão umbilical de recém-nascidos prétermo de muito baixo peso. J Pediatr. 2012;88(1):61-6. doi: 10.2223/JPED.2158.

3. Barbosa KBF, Costa NMB, Alfenas RG, De Paula SO, Minim VPR, Bressan J. Estresse oxidativo: conceito, implicações e fatores modulatórios. Rev Nutr. 2010;23(4):629-43. doi: 10.1590/S1415-52732010000400013.

4. Arad I, Bar-Oz B, EZ NA, Barak V. Interleukin-6 and $\mathrm{N}$-terminal pro-brain natriuretic peptide cord blood levels in premature infants: correlations with perinatal variables. Isr Med Assoc J. 2010;12(7):419-23. PMID: 20862823.

5. Fagundes DJ, Taha MO. Modelo animal de doença: critérios de escolha e espécies de animais de uso corrente. Acta Cir Bras. 2004;19(1):59-65. doi: 10.1590/S010286502004000100010.

6. Schanaider A, Silva PC. Uso de animais em cirurgia experimental. Uso de animais em cirurgia experimental. Acta Cir Bras. 2004;19(4):441-7.doi: 10.1590/S010286502004000400014.

7. Schneider CD, Oliveira AR. Radicais livres de oxigênio e exercício: mecanismos de formação e adaptação ao treinamento físico. Rev Bras Med Esporte. 2004;10(4):308-13. doi: 10.1590/S1517-86922004000400008.

8. Lykkesleldt J. Malondialdehyde as biomarker of oxidative damage to lipids caused by smoking. Clin Chim Acta. 2007;380(1-2):508. doi: 10.1016/j.cca.2007.01.028.

9. Groen B, Uuldriks GA, de Vos P, Visser JT, Links TP, Faas MM. Impaired trophoblast 
invasion and increased numbers of immune cells at day 18 of pregnancy in the mesometrial triangle of type 1 diabetic rats. Placenta. 2015;36(2):142-9. doi: 10.1016/j. placenta.2014.12.004.

10.Wietzycoski CR, Marchesini JCD, Al-Themyat $\mathrm{S}$, Meyer FS, Trindade MRM. Improvement in oxidative stress after duodenojejunostomy in an experimental model of type 2 diabetes mellitus. Arq Bras Cir Dig. 2016;29(Supl.1):37 doi: 10.1590/0102-6720201600S10002.

11. Halliwell B, Whiteman M. Measuring reactive species and oxidative damage in vivo and in cell culture: how should you do it and what do the results mean? $\mathrm{Br}$ J Pharmacol. 2004;142(2):231-55. doi: 10.1038/sj.bjp.0705776.

12.Caldas JPS, Vilela MS, Braghini CA, Mazzola TN, Marba STM. Uso materno antenatal de corticosteroide e marcadores de estresse oxidativo e de inflamação no sangue de cordão umbilical de recém-nascidos prétermo de muito baixo peso. J Pediatr. 2012;88(1):61-6. doi: 10.2223/JPED.2158.

13.Dotan $Y$, Lichtenberg D, Pinchuk L. Lipid peroxidation cannot be used as a universal criterion of oxidative stress. Prog Lipid Res. 2004;43(3):200-27. doi: 10.1016/2003.10.001.

14.Posada D. jModelTest: phylogenetic model averaging. Mol Biol Evol. 2008;25(7):12536. doi: $10.1093 / \mathrm{molbev} / \mathrm{msn} 083$.

15.Nikki C, Tatsuya O, Massimo S, AnneMarie $\mathrm{B}$, Cameron WB, Dolores $\mathrm{H}$, Eric $\mathrm{CH}$. Perivascular nitric oxide activates notch signaling and promotes stem-like character in PDGF-induced glioma cells. Cell Stem Cell. 2010;6(2):141-52 doi: 10.1016/j. stem.2010.01.001.

16.Souza Jr. TP, Oliveira PR, Pereira B. Exercício físico e estresse oxidativo: efeitos do exercício físico intenso sobre a quimioluminescência urinária e malondialdeído plasmático. Rev Bras Med Esporte. 2005;11(1):91-6. doi: 10.1590/S1517-86922005000100010.

17.Silveira SL. Programação metabólica: estudo de parâmetros indicadores de resistência à insulina e espécies reativas de oxigênio em ratos [Tese]. Instituto de Ciências Básicas da Saúde, Universidade Federal do Rio Grande do Sul; 2010.

18. Martim AC, Sanders RA, Watkins JB. Diabetes, oxidative stress, and antioxidants: a review.
J Biochem Mol Toxicol. 2003;17(1):24-38. doi: 10.1002/10058.

19. Kawamura M, Heinecke JW, Chait A. Pathophysiological concentrations of glucose promote oxidative modification of low density lipoprotein by a superoxidedependent pathway. J Clin Invest. 1994;94(2):771-8. doi: 10.1172/ JCl117396.

20.Nédic O, Rattan SIS, Grune T, Trougakos IP. Molecular effects of advanced glycation end products on cell signalling pathways, ageing and pathophysiology. Free Radic Res. 2013;47(Suppl 1):28-38. doi: 10.3109/10715762.2013.806798.

21.Baynes JW, Thorpe SR. Role of oxidative stress in diabetic complications: a new perspective on an old paradigm. Diabetes. 1999;48(1):19. doi: 10.2337/diabetes.48.1.1.

22. Mohamed AK, Bierhaus A, Schiekofer S, Tritschler H, Ziegler R, Nawroth PP. The role of oxidative stress and NF-kappaB activation in late diabetic complications. Biofactors. 1999;10(2-3):157-67. PMID: 10609877.

23.Singh R, Kaur N, Kishore L, Kumar GG. Management of diabetic complications: $A$ chemical constituents based approach. J Ethnopharmacol. 2013;150(1):51-70. doi: 10.1016/j.jep.2013.08.051.

24.Buonocore $G$, Perrone $S$, Longini $M$, Vezzosi $P$, Marzocchi B, Paffetti P, Bracci R. Oxidative stress in preterm neonates at birth and on the seventh day of life. Pediatr Res. 2002;52:46-9. doi: 10.1203/00006450200207000-00010.

25.Mulay S, Philip A, Solomon S. Influence of maternal diabetes on fetal rat development: Alteration of insulin receptors in fetal liver and lung. J. Endocrinol. 1983;98(3):401-10. doi: $10.1677 /$ joe.0.0980401.

26. Merzouk H, Madani S, Sari DC, Prost J, Bouchenak M, Belleville J. Time course of changes in serum glucose, insulin, lipids and tissue lipase activities in macrosomic offspring of rats with streptozotocininduced diabetes. Clin Sci. 2000;98(1):2130. doi: $10.1042 / \operatorname{cs} 0980021$.

27.Pimentel AML, Pereira NR, Costa CA, Mann GE, Cordeiro VSC, Moura RS, Brunini TMC, Mendes-Ribeiro AC, Resende AC. L-argininenitric oxide pathway and oxidative stress in plasma and platelets of patients with preeclampsia. Hypertens Res. 2013;36(9):7838. doi: 10.1038/hr.2013.34. 
28.Calabrese V, Cornelius C, Dinkova-Kostova AT, Calabrese EJ, Mattson MP. Cellular stress responses, the hormesis paradigm, and vitagenes: novel targets for therapeutic intervention in neurodegenerative disorders. Antioxid Redox Signal. 2010;13(11):1763811.doi: 10.1089/ars.2009.3074.

29.Gracy X. Rosario RA, Toshihiro K, Michael JS. Intrauterine fate of invasive trophoblast cells. Placenta. 2009;30(5):457-63. doi:
10.1016/2009.02.008.

30.Lamb SE, Hansen Z, Lall R, Castelnuovo E, Withers EJ, Nichols V, Potter R, Underwood MR; Back Skills Training Trial investigators. Group cognitive behavioural treatment for low-back pain in primary care: a randomised controlled trial and cost-effectiveness analysis. Lancet. 2010;375 916-23. doi: 10.1016/S0140-6736(09)62164-4.

\section{Correspondence:}

Ricardo Dutra Aydos

Estrada EW2, 194

79037-802 Campo Grande - MS Brasil

Tel.: (55 67)99984-5773

ricardoaydos@gmail.com

Received: May 06, 2018

Review: July 09, 2018

Accepted: Aug 04, 2018
Conflict of interest: none

Financial sources: FINEP, FAPEC, and FUNDECT

${ }^{1}$ Research performed at Laboratory of Experimental Carcinogenesis and Cancer Studies, Universidade Federal do Mato Grosso do Sul (UFMS), Campo Grande-MS, Brazil. 